\title{
INVERSE PROBLEMS IN AHP
}

\author{
Masaaki Shinohara* \\ Nihon University \\ Izumi-chou, Narashino \\ Chiba 275-8575, Japan
}

\begin{abstract}
Two classes of inverse problems in the process of the AHP are studied. The first class, which we call "inverse hierarchy problem", is the inverse of the forward problem of obtaining the overall priority weight vector for the alternative set from the local priority weight data set, such as the weight vectors for criteria viewed from the goal and for alternatives viewed from each criterion. The second class, which we call "inverse eigenvector problem", is the inverse of the forward problem of obtaining the eigenvector from the pairwise comparison matrix, where complete information of pairwise comparison data is assumed.

For the inverse hierarchy problem, two types of the problems are mathematically formulated as constrained least $p$-th norm problems. The inverse hierarchy problem of type 1 is to estimate, from the given objective overall priority weight vector for the alternative set, the local priority weight vector for the criteria. The inverse hierarchy problem of type 2 is to estimate, from the given objective overall priority weight vector for the alternative set, some of the local priority weight vectors for the alternatives viewed from each criterion.

For the inverse eigenvector problem, we show that, given a priority weight vector $\boldsymbol{x}$ and an eigenvalue $\lambda$ (or Consistency Index $C I=(\lambda-N) /(N-1)$ ), the problem of finding an $N \times N$ matrix $A$ which satisfies $A \boldsymbol{x}=\lambda \boldsymbol{x}$ can be equivalently transformed into the problem of finding an $N \times N$ matrix $E$ which satisfies $E \mathbf{I}=\lambda \mathbf{1}$, where $e_{i j}=a_{i j}\left(x_{j} / x_{i}\right), \boldsymbol{x}>\mathbf{0}$, and $\boldsymbol{1}$ is the all- 1 column vector. For $\mathrm{N}=3$, the reciprocity assumed and $\lambda$ given, the error matrix $E$ is determined uniquely in the sense that a quadratic equation has a unique pair of solutions. On the basis of this result for $\mathrm{N}=3$, the inverse eigenvector problem " $E \boldsymbol{I}=\lambda \mathbf{1}$ " is analyzed for cases of $N=3 m, 3 m+1$, and $3 m+2$, respectively, and the error matrix $E$ is shown to be expressed explicitly with $N(N-3) / 2$ free independent parameters.
\end{abstract}

Keywords: inverse problem, eigenvector, eigenvalue, Consistency Index, quadratic equation, reciprocal error matrix, reciprocity

\section{Introduction}

Two classes of inverse problems in the process of the AHP are studied. The first class, which we call "inverse hierarchy problem", is the inverse of the forward problem of obtaining the overall priority weight vector for the alternative set from the local priority weight data set, such as the weight vector for criteria viewed from the goal and the weight vectors for alternatives viewed from each criterion. The second class, which we call "inverse eigenvector problem", is the inverse of the forward problem of obtaining the eigenvector from the

${ }^{*}$ Corresponding author 
pairwise comparison matrix or the pairwise comparison data set.

In Chapter 2, these two classes of AHP inverse problems, the inverse hierarchy problem and the inverse eigenvector problem, are explained.

In Chapter 3, two types of the inverse hierarchy problems are discussed and are formulated as constrained least square problems.

In Chapter 4, the inverse eigenvector problem of finding an $N \times N$ pairwise comparison matrix $A$ satisfying $A \boldsymbol{x}=\lambda \boldsymbol{x}$ with given $\boldsymbol{x}$ and $\lambda$, which is equivalent to the problem of finding an $N \times N$ error matrix $E$ which satisfies $E \mathbf{l}=\lambda \boldsymbol{I}$ with given $\lambda$, is analyzed, first for $\mathrm{N}=3$, second for $\mathrm{N}=4,5$ and 6 , and finally for cases of $N=3 m, 3 m+1$, and $3 m+2$, respectively.

\section{Two classes of AHP inverse problems}

The forward, or direct, process of AHP consists of following four steps.

Step 1: Model the problem as a hierarchy (Decomposition).

Step 2: Make pairwise comparisons between elements, such as between criteria viewed from the goal and between alternatives viewed from each criterion (Pairwise comparison).

Step 3: Estimate the local priority weight vector, such as the weight vectors for criteria viewed from the goal and for alternatives viewed from each criterion, as an eigenvector of pairwise comparison matrix (Eigenvector).

Step 4: Synthesize these local priority weight data along the hierarchy into the overall priority weight vector for the alternatives (Synthesis).

Two classes of inverse problems are presented in the above procedure of the AHP. The first class, which we call "inverse hierarchy problem", is the inverse of the forward problem of Step 4 (Synthesis). The second class, which we call "inverse eigenvector problem", is the inverse of the forward problem of Step 3 (Eigenvector).

\section{Inverse hierarchy problem}

Two types of inverse hierarchy problems are mathematically formulated as constrained least $p$-th norm problems. The inverse hierarchy problem of type 1 is to estimate the local priority weight vector for the criteria from the given objective overall priority weight vector for the alternative set. The inverse hierarchy problem of type 2 is to estimate some of the local priority weight vectors for the alternatives viewed from each criterion from the given objective overall priority weight vector for the alternative set.

\subsection{Inverse hierarchy problem of type 1}

The inverse hierarchy problem of type 1 is to estimate, $\boldsymbol{x}=\left\{x_{j}\right\}, m \times 1$ local priority weight vector for the criteria, from, $\boldsymbol{y}=\left\{y_{i}\right\}, n \times 1$ given objective overall priority weight vector for the alternative set. Its forward problem is " $y=D x$ (1)", where $D=\left\{d_{i j}\right\}$ is $n \times m$ evaluation matrix between $n$ alternatives and $m$ criteria. This problem is generally formulated as the $p$-th norm distance minimization problem with normalization and non-negativity constraints.

Objective: $Z_{l}=\| \boldsymbol{y}$-Dx $\|_{p} \rightarrow$ minimize

Normalization constraint: $\Sigma x_{j}=1$

Non-negative variable: $\boldsymbol{x} \geqq 0$

Let $p=2$, and consider the equality-constrained minimization problem. Objective: $Z_{l}=\left(\boldsymbol{y}^{T}-\boldsymbol{x}^{T} \boldsymbol{D}^{T}\right)(\boldsymbol{y}-\boldsymbol{D} \boldsymbol{x}) \rightarrow$ minimize

Normalization constraint: $\Sigma x_{j}=1$

In solving the original problem (2)-(4), we take a recursive approach: first solve an equalityconstrained problem, such as (5) and (6), and if its solution does not satisfy the inequality constraint (4), then solve equality-constrained problems of smaller size. This procedure is iterated until the solution of the original problem is obtained. 


\subsection{Inverse hierarchy problem of type 2}

The inverse hierarchy problem of type 2 is to estimate $\boldsymbol{b}$, a part of $\boldsymbol{D}=\left\{d_{i j}\right\}$, from the rest of $\boldsymbol{D}$, $\boldsymbol{x}=\left\{x_{j}\right\}$ and $\boldsymbol{y}=\left\{y_{i}\right\}$. For the case $p=2$ and $\boldsymbol{b}$ is the $m$-th column of $\boldsymbol{D}$, the problem is formulated as follows.

Objective: $Z_{2}=\left(\boldsymbol{b}^{T}-c^{T}\right)(\boldsymbol{b}-\boldsymbol{c}) \rightarrow$ minimize

Normalization constraint: $\Sigma b_{j}=1$

Non-negative variable: $\boldsymbol{b} \geqq \mathbf{0}$

$\left.\boldsymbol{D}=(\boldsymbol{B}, \boldsymbol{b}), \boldsymbol{y}=\boldsymbol{D} \boldsymbol{x}=(\boldsymbol{B}, \boldsymbol{b}) \mathrm{C}_{\mathrm{Z}}^{\mathrm{V}}\right)=\boldsymbol{B} \boldsymbol{v}+\boldsymbol{b} \boldsymbol{z}$, and $\boldsymbol{c}=(\boldsymbol{y}-\boldsymbol{B} \boldsymbol{v}) / \boldsymbol{z}$, where $\boldsymbol{b}$ is a variable vector and the rest are given. Since the objective function (7) is quadratic and spherical, if the solution of an equality-constrained problem, such as (7) and (8), does not satisfy the inequality-constraint (9), then we only have to solve one equality-constrained problem of smaller size with all negative $b_{j}$ 's being fixed at $O$ 's.

\section{Inverse eigenvector problem}

Given a priority weight vector $\boldsymbol{x}$ and an eigenvalue $\lambda$ (or Consistency Index $C I=(\lambda-N) /(N-1)$ ), the problem of finding an $N \times N$ pairwise comparison matrix $A$ which satisfies $A \boldsymbol{x}=\lambda \boldsymbol{x}$ is equivalent to the problem of finding an $N \times N$ error matrix $E$ which satisfies $E \boldsymbol{I}=\lambda \mathbf{1}$, where $e_{i j}=a_{i j}\left(x_{j} / x_{i}\right), \boldsymbol{x}>\mathbf{0}$, and $\boldsymbol{1}$ is the all- 1 column vector.

\subsection{Case of $N=3$}

Assuming the reciprocity of $E=\left\{e_{i j}\right\}$, the problem of finding $E$ which satisfies $E \mathbf{l}=\lambda \mathbf{1}$ is expressed by a set of nonlinear equations (10), (11) and (12).

$$
\begin{aligned}
e_{12}+e_{13} & =K_{1} \\
+e_{23} & =K_{2} \\
1 / e_{12} & =K_{3}
\end{aligned}
$$

Although $K_{1}=K_{2}=K_{3}=k=\lambda-1$ for $N=3$ (13), we introduced $K_{1}, K_{2}$ and $K_{3}$ to prepare for further analysis. Using the quadratic formula, $e_{12}$ is given by (14), and consequently, $e_{13}$ and $e_{23}$ are also derived.

$$
e_{12}=\frac{1}{2\left(K_{2} K_{3}-1\right)}\left\{-\left[K_{2}-K_{3}-K_{1}\left(K_{2} K_{3}-1\right)\right] \pm \sqrt{\left[K_{2}-K_{3}-K_{1}\left(K_{2} K_{3}-1\right)\right]^{2}-4\left(K_{1} K_{3}-1\right)\left(K_{2} K_{3}-1\right)}\right\}
$$

Since (13) holds for $N=3$, we have two patterns of error matrix, $E^{+}$and $E^{-}$, given by (15).

$e_{12}^{+}=\frac{1}{2}\left(k \pm \sqrt{k^{2}-4}\right)(=e), e_{12}^{-}=e^{-1}, e_{13}{ }^{+}=e^{-1}, e_{13}^{-}=e, e_{23}{ }^{+}=e, e_{23}^{-}=e^{-1}$

\subsection{Case of $N=4$}

The nonlinear equation with variables $e_{12}, e_{13}$ and $e_{23}$ is solved by setting $K_{1}, K_{2}$ and $K_{3}$ as in (16), and using the quadratic formula, $e_{12}$ is given by (14) and consequently, $e_{13}$ and $e_{23}$ are derived.

$K_{1}=\lambda-1-e_{14}, K_{2}=\lambda-1-e_{24}, K_{3}=\lambda-1-e_{34}$

$$
e_{14}=1 /\left(\lambda-1-1 / e_{24}-1 / e_{34}\right)
$$

Since $e_{14}$ is determined by (17) if $e_{24}$ and $e_{34}$ are given, when two free parameters, $e_{24}$ and $e_{34}$, are given, the error matrix $E$ is determined uniquely in the sense that a quadratic equation has a unique pair of solutions.

\subsection{Case of $N=5$}

The nonlinear equation with variables $e_{12}, e_{13}$ and $e_{23}$ is solved by setting $K_{l}, K_{2}$ and $K_{3}$ as in (18), and using the quadratic formula, $e_{12}$ is given by (14) and consequently, $e_{13}$ and $e_{23}$ are derived.

$$
\begin{aligned}
& K_{1}=\lambda-1-e_{14}-e_{15}, K_{2}=\lambda-1-e_{24}-e_{25}, K_{3}=\lambda-1-e_{34}-e_{35} \quad(18) \\
& e_{14}=1 /\left(\lambda-1-1 / e_{24}-1 / e_{34}-e_{45}\right) \quad(19) \quad e_{45}=1 /\left(\lambda-1-1 / e_{15}-1 / e_{25}-1 / e_{35}\right)
\end{aligned}
$$

Since $e_{14}$ is determined by (19) if $e_{24}, e_{34}$ and $e_{45}$ are given and $e_{45}$ is determined by (20) if $e_{15}$, $e_{25}$ and $e_{35}$ are given, when five free parameters, $e_{15}, e_{24}, e_{25}, e_{34}$ and $e_{35}$, are given, the error 
matrix $E$ is determined uniquely.

\subsection{Case of $N=6$}

We have two sets of nonlinear equations in case $N=6$. While one nonlinear equation with variables $e_{12}, e_{13}$ and $e_{23}$ is solved by setting $K_{1}, K_{2}$ and $K_{3}$ as in (21), and $e_{12}$ is given by (14) and consequently, $e_{13}$ and $e_{23}$ are derived, the other nonlinear equation with variables $e_{45}, e_{46}$ and $e_{56}$ is solved by setting $K_{1}, K_{2}$ and $K_{3}$ as in (22), and $e_{45}$ is given by (14) and consequently, $e_{46}$ and $e_{56}$ are derived.

$K_{1}=\lambda-1-e_{14}-e_{15}-e_{16}, \quad K_{2}=\lambda-1-e_{24}-e_{25}-e_{26}, \quad K_{3}=\lambda-1-e_{34}-e_{35}-e_{36}$

$K_{1}=\lambda-1-1 / e_{14}-1 / e_{24}-1 / e_{34}, \quad K_{2}=\lambda-1-1 / e_{15}-1 / e_{25}-1 / e_{35} \quad K_{3}=\lambda-1-1 / e_{16}-1 / e_{26}-1 / e_{36}$

When nine free parameters, $e_{14}, e_{15}, e_{16}, e_{24}, e_{25}, e_{26}, e_{34}, e_{35}$ and $e_{36}$, are given, the error matrix $E$ is determined uniquely in the sense that a quadratic equation has a unique pair of solutions, or namely, we have four patterns of error matrix, $E^{++}, E^{+-}, E^{-+}$and $E^{--}$.

\subsection{General case of $N$}

Assuming the reciprocity of $E$, the nonlinear equation " $E \boldsymbol{I}=\lambda \boldsymbol{l}$ " has $U$ variables, $Q$ equations, and $F$ free independent variables, or parameters.

$U=N(N-1) / 2, \quad Q=N, \quad F=N(N-3) / 2, \quad U=Q+F \quad(23)$

Here, $Q=$ the number of dependent variables, and $F=$ the degree of freedom.

The regions corresponding $U$ variables and $F$ free parameters in the error matrix $E$ will be illustrated for the cases of $N=3 m, 3 m+1$, and $3 m+2$, respectively.

[Case of $\mathbf{N = 3 m}$ ] The error matrix $E$ is shown in Figure 1(a), where its upper triangular part excluding the diagonal elements corresponds to $U$ variables $(U=N(N-1) / 2)$ and its shadowed part corresponds to $F$ free parameters. The rest, $U-F$, corresponds to $Q$ equations, or $Q$ dependent variables denoted by $\bigcirc$.

Given $F$ free parameters in the shadowed part, $Q(=3 m)$ dependent variables in the diagonal blocks are determined uniquely in the sense that a quadratic equation has a unique pair of solutions, by solving $m$ quadratic equations(three dependent variables in a diagonal block by solving one quadratic equation), or namely, we have $2^{m}$ patterns of error matrix.

[Case of $N=3 m+1$ ] The error matrix $E$ is shown in Figure 1(b), where its upper triangular part excluding the diagonal elements corresponds to $U$ variables and its shadowed part corresponds to $F$ free parameters. The rest, $U-F$, corresponds to $Q$ equations, or $Q$ dependent variables denoted by $\bigcirc$ and $\bullet$, where $e_{I N}$, denoted by $\bullet$, is chosen as a dependent variable, following the manner $e_{14}$ is chosen as a dependent variable as in (17) for case $\mathrm{N}=4$.

Given $F$ free parameters in the shadowed part, $3 m$ dependent variables(out of $Q=3 m+1$ dependent variables) in the diagonal blocks denoted by $\bigcirc$ are determined uniquely with $2^{m}$ patterns, and the remaining one dependent variable $e_{1 N}$ denoted by $\bullet$ is determined by (24).

$e_{1 N}=1 /\left(\lambda-1-1 / e_{2 N^{-}}-1 / e_{3 N^{-}}-1 / e_{4 N^{-}}-1 / e_{5 N} \ldots \ldots-1 / e_{N-2, N^{-}}-1 / e_{N-1, N}\right)$

[Case of $N=3 m+2$ ] The error matrix $E$ is shown in Figure 1(c), where its upper triangular part excluding the diagonal elements corresponds to $U$ variables and its shadowed part corresponds to $F$ free parameters. The rest, $U-F$, corresponds to $Q$ equations, or $Q$ dependent variables denoted by $\bigcirc, \bullet$ and $\boldsymbol{\Lambda}$, where $e_{1, N-1}$, denoted by $\bullet$, is chosen as a dependent variable, following the manner $e_{14}$ is chosen as a dependent variable as in (19) for case $\mathrm{N}=5$ and $e_{N-1, N}$, denoted by $\boldsymbol{\Lambda}$, is chosen as a dependent variable, following the manner $e_{45}$ is chosen as a dependent variable as in (20) for case $\mathrm{N}=5$. Given $F$ free parameters in the shadowed part, $3 m$ dependent variables(out of $Q=3 m+2$ dependent variables) in the diagonal blocks denoted by $\bigcirc$ are determined uniquely with $2^{m}$ patterns, and remaining one dependent variable $e_{1, N-I}$ denoted by $\bullet$ is determined by (25) and remaining the other dependent variable $e_{N-1, N}$ denoted by $\boldsymbol{\Lambda}$ is determined by (26).

$e_{1, N-1}=1 /\left(\lambda-1-1 / e_{2, N-1}-1 / e_{3, N-1}-1 / e_{4, N-1} \ldots \ldots-1 / e_{N-2, N-1}-e_{N-1, N}\right)$

$e_{N-1, N}=1 /\left(\lambda-1-1 / e_{1 N^{-}}-1 / e_{2 N^{-}}-1 / e_{3 N^{-}}-1 / e_{4 N} \ldots \ldots-1 / e_{N-3, N^{-}} 1 / e_{N-2, N}\right)$

International Symposium of 4

the Analytic Hierarchy

Washington, D. C. Process June 29 - July 2, 2014 


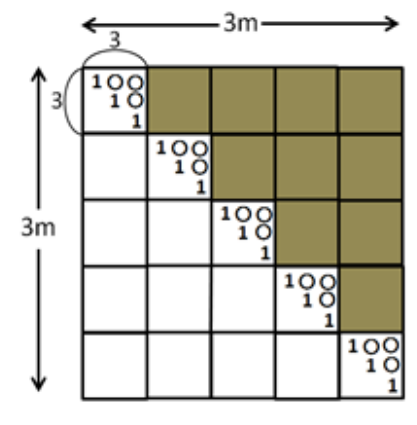

(a) $N=3 m$

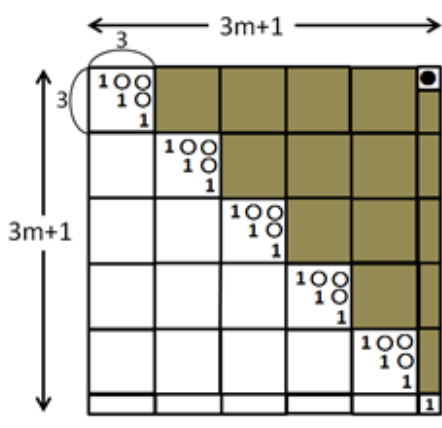

(b) $N=3 m+1$

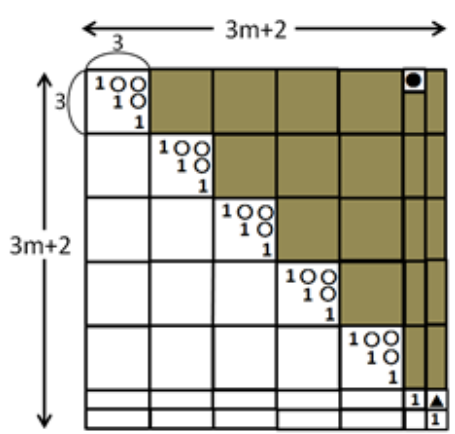

(c) $N=3 m+2$

Figure 1: Error matrixes for cases of $N=3 m, 3 m+1$, and $3 m+2$

\section{Conclusions}

Two classes of AHP inverse problems, inverse hierarchy problem and inverse eigenvector problem, are proposed, formulated, and analyzed.

In solving the two types of inverse hierarchy problems, we employed a recursive approach where, first, negative weights are allowed to minimize the error between the goal and the achieved by relaxing the non-negativity constraints, and then, the solution is adjusted by fixing the negative weights at $O$ 's and solving equality-constrained problems of smaller size. This approach is supposed to simulate our way of thinking: we keep things of positive value and discard things of negative value. This recursive approach is mathematically called "Visible Region Approach(Shinohara, 1994)," because the optimum of a constrained minimization problem is assured to lie on the surface of the feasible region which is visible from an optimum of the associated unconstrained minimization problem, if the objective function is convex. Some of the application examples are Japanese capital relocation and handicapped match of horse race (Takai \& Shinohara, 2013, Takai, 2014), where with handicapped match of horse race an additional burden (=handicap) is imposed on each horse so that winning chance of every horse is equalized as much as possible to make it exciting.

The inverse eigenvector problem $A \boldsymbol{x}=\lambda \boldsymbol{x}$, or $E \boldsymbol{I}=\lambda \mathbf{1}$, is to solve a set of nonlinear equations, resulting in $m$ quadratic equations for the cases of $N=3 m, 3 m+1$, and $3 m+2$, and, if $F$ free independent parameters are given $(F=N(N-3) / 2)$, the error matrix $E$ is determined uniquely with $2^{m}$ patterns. The inverse eigenvector problem is expected to find applications in the field of opinion manipulation, such as controlling $a_{i j}$ 's to achieve objective priority weight and/or objective CI.

\section{References}

Saaty, T.L. (1980). The Analytic Hierarchy Process, McGraw-Hill, New York.

Shinohara, Masaaki (1994). Parameter estimation by nonlinear least square method (in Japanese), Joint Research Report of the Institute of Statistical Mathematics 61, Modelling and algorithm for optimization 5, pp.216-241(1994.2)

Takai, Ryousuke, \& Masaaki Shinohara (2013). AHP Inverse Problem and its Application to the Capital Function Relocation (in Japanese), Proceedings of the 46th Annual Conference of College of Industrial Technology, Nihon University, 2-43 (2013.12.7).

Takai, Ryousuke (2014). Study on AHP Inverse Problem, Master Thesis of College of Industrial Technology, Nihon University (2014. 3) 